\title{
Molecular surveillance of carbapenemase- producing Pseudomonas aeruginosa at three medical centres in Cologne, Germany
}

\author{
Elena Schäfer ${ }^{1 \dagger}$, Monika Malecki ${ }^{1 \dagger}$, Carlos J. Tellez-Castillo ${ }^{2}$, Niels Pfennigwerth ${ }^{3}$, Lennart Marlinghaus ${ }^{3}$,
} Paul G. Higgins ${ }^{4} \mathbb{D}$, Frauke Mattner ${ }^{1}$ and Andreas F. Wendel ${ }^{1^{*}}$

\begin{abstract}
Background: Pseudomonas aeruginosa is a common pathogen causing hospital-acquired infections. Carbapenem resistance in $P$. aeruginosa is either mediated via a combination of efflux pumps, AmpC overexpression, and porin loss, or through an acquired carbapenemase. Carbapenemase-producing P. aeruginosa (CPPA) strains are known to cause outbreaks and harbour a reservoir of mobile antibiotic resistance genes, however, few molecular surveillance data is available. The aim of this study was to analyse the prevalence and epidemiology of CPPA in three German medical centres from 2015 to 2017.
\end{abstract}

Methods: Identification and susceptibility testing were performed with VITEK 2 system. P. aeruginosa nonsusceptible to piperacillin, ceftazidime, cefepime, imipenem, meropenem and ciprofloxacin (4MRGN according to the German classification guideline) isolated from 2015 to 2017 were analysed. A two-step algorithm to detect carbapenemases was performed: phenotypic tests (EDTA- and cloxacillin-combined disk tests) followed by PCR, Sanger sequencing, and eventually whole genome sequencing. CPPA isolates were further genotyped by RAPD and PFGE. In-hospital transmission was investigated using conventional epidemiology.

Results: Sixty two P. aeruginosa isolates were available for further analysis, of which 21 were CPPA as follows: bla $a_{\mathrm{VM}-1}$ $(n=2)$, bla $a_{\mathrm{VIM}-2}(n=17)$, bla $a_{\mathrm{NDM}-1} / b_{1} a_{\mathrm{GES}-5}(n=1)$ and the newly described bla $a_{\mathrm{MP}-82}(\mathrm{n}=1)$. CPPA were mostly hospitalacquired (71.4\%) and isolated on intensive care units (66.7\%). All (except one) were from the tertiary care centre. PFGE typing revealed one large cluster of VIM-2-producing CPPA containing 13 isolates. However, using conventional epidemiology, we were only able to confirm three patient-to-patient transmissions, and one room-to-patient transmission, on several intensive care units.

Conclusions: These data give insight into the epidemiology of CPPA in three centres in Germany over a period of 3 years. Carbapenemases are a relevant resistance mechanism in 4MRGN-P. aeruginosa, illustrated by genetically related VIM-2-producing strains that seem to be endemic in this region. Our data suggest that infection control measures should especially focus on controlling transmission on the ICU and support the need for a local molecular surveillance system.

Keywords: Pseudomonas aeruginosa, Carbapenemase, Surveillance, VIM-2

\footnotetext{
* Correspondence: wendela@kliniken-koeln.de

${ }^{\dagger}$ Elena Schäfer and Monika Malecki contributed equally to this work.

${ }^{1}$ Institute of Hygiene, Cologne Merheim Medical Centre, University Hospital

of Witten/Herdecke, Ostmerheimer Strasse 200, 51109 Cologne, Germany

Full list of author information is available at the end of the article
}

(c) The Author(s). 2019 Open Access This article is distributed under the terms of the Creative Commons Attribution 4.0 International License (http://creativecommons.org/licenses/by/4.0/), which permits unrestricted use, distribution, and reproduction in any medium, provided you give appropriate credit to the original author(s) and the source, provide a link to the Creative Commons license, and indicate if changes were made. The Creative Commons Public Domain Dedication waiver (http://creativecommons.org/publicdomain/zero/1.0/) applies to the data made available in this article, unless otherwise stated. 


\section{Background}

Pseudomonas aeruginosa is a leading nosocomial pathogen and infections can be difficult to treat because of rapid resistance development. The emergence of multidrug-resistant (MDR) isolates is a serious public health threat and often affects immunocompromised patients within special units (intensive care units (ICU), haematology-oncology wards or burn units) [1-4]. Resistance to carbapenems is mediated either by intrinsic resistant mechanisms (a combination of efflux pumps, AmpC overexpression and porin loss) or acquisition of a carbapenemase, especially a metallo- $\beta$ lactamase (MBL) [5]. Carbapenemase-producing P. aeruginosa (CPPA) isolates harbour antimicrobial resistance genes located on mobile genetic elements (mainly integrons, transposons or plasmids) that can spread to other bacteria [6-8], so microbiological monitoring and infection control surveillance is of utmost importance. Prevalence of CPPA among MDR $P$. aeruginosa differs greatly between regions, with VIM- and IMP-family carbapenemases being the most widespread $[9,10]$. Additionally, CPPA are known to cause protracted outbreaks, e.g. IMP-8 or GIM-1-producing types $[11,12]$. However, there is little surveillance data available combining molecular and epidemiological information. The aim of this study was to analyse the prevalence and epidemiology of CPPA in three German medical centres isolated from 2015 to 2017.

\section{Methods}

\section{Setting and screening strategy}

The Institute of Hygiene at the Cologne Merheim Medical Centre provides an infection control service for three medical centres in Cologne (one tertiary care centre, 700 beds; one secondary care centre, 400 beds; one children hospital, 260 beds) with a total of seven ICUs between them. Microbiological specimens are sent to the private microbiology laboratory MVZ synlab Leverkusen. The protocol of the German healthcare-associated infection surveillance on intensive care units (ITS-KISS) was followed on all seven ICUs during the study period [13]. The number of patients colonized/infected with MDR $P$. aeruginosa was assessed using the laboratory surveillance information system (Hybase v.6, epiNET AG, Germany). A risk-based rectal admission screening on multidrug-resistant Gram-negative organisms was performed in the three hospitals (stay at a healthcare facility abroad or on a German ICU within the last year, known positive carrier status or contact to other patients carrying carbapenem-resistant Gram-negative organisms). On most intensive care units (five out of seven) a general admission screening was implemented.

\section{Identification and susceptibility testing}

All inpatient isolates were identified with standard microbiological procedures using the VITEK 2 system (Vitek GN-ID, bioMérieux, Marcy l'Etoile, France) or
MALDI-TOF (Bruker Daltonics, Bremen, Germany). Susceptibility testing was performed with the VITEK 2 system (Vitek AST-N248). EUCAST breakpoints were used for interpretation (v.8.0, May 2018). P. aeruginosa non-susceptible (intermediate or resistant) to piperacillin, ceftazidime, cefepime, imipenem, meropenem and ciprofloxacin (4MRGN according to the German classification guideline for Gram-negative multidrug-resistant organisms [14], at least MDR according to ECDC/CDC classification [15]) isolated from clinical and screening specimens from 2015 to 2017 were included. Bacterial isolates were stored in a $30 \%$-glycerol stock at $-20^{\circ} \mathrm{C}$.

\section{Phenotypic and molecular screening and detection of carbapenemases}

A two-step algorithm to detect carbapenemases was performed, comprised of phenotypic and genotypic tests. We performed two combined disk tests (CDT) using (a) $10 \mu \mathrm{g}$ imipenem with or without $930 \mu \mathrm{g}$ EDTA and (b) $10 \mu \mathrm{g}$ imipenem with or without $4000 \mu \mathrm{g}$ cloxacillin. A difference of (a) $\geq 5 \mathrm{~mm}$ or (b) $<6 \mathrm{~mm}$ in zone diameter was considered to be indicative of (a) an MBL [16] or (b) a carbapenemase [17]. Quality controls with strains provided by the German National Reference Centre for Multidrugresistant Gram-negative Bacteria were performed. CDTpositive isolates were further confirmed by several PCRs and sequencing, first a $b l a_{\mathrm{IMP}} / b l a_{\mathrm{VIM}}$ duplex PCR $[16,18]$, followed by screening for the $b l a_{\mathrm{GIM}-1}, b l a_{\mathrm{NDM}}, b l a_{\mathrm{KPC}}$, $b l a_{\mathrm{OXA}-48}$ and $b l a_{\mathrm{GES}}$ genes $[6,19]$.

One IMP-producing isolate was further examined by whole genome sequencing because we were unable to assess the exact $b l a_{\mathrm{IMP}}$-type by conventional sequencing. Total DNA was isolated using the MagAttract HMW DNA Kit (Qiagen, Hilden, Germany). Sequencing libraries were prepared using the Nextera XT library prep kit (Illumina $\mathrm{GmbH}$, Munich, Germany) for a $250 \mathrm{bp}$ paired-end sequencing run on an Illumina MiSeq sequencer. De novo assembly was performed using Velvet (version 1.1.04) [20]. An N50 of 52,548 bp was achieved. Acquired resistance genes on assembled sequences were identified by ResFinder (version 3.1; threshold of $98 \%$ identity and minimum length of $60 \%$ ) [21]. Sequence reads of the newly described $b l a_{\text {IMP-82- }}$ variant have been deposited under the nucleotide accession number GenBank MN057782.

\section{Genotyping}

Carbapenemase-positive isolates were first genotyped by RAPD (three primers: ERIC-1, ERIC-2 and ST272 [22]). Isolates differing by one or more bands were assigned to distinct types. Genotyping was additionally carried out by PFGE after BculI/SpeI (New England BioLabs, USA) restriction under the following conditions: $6 \mathrm{~V} / \mathrm{cm}$ for 
$24 \mathrm{~h}$ with pulse times of $5 \mathrm{~s}$ to $33 \mathrm{~s}$ at $14^{\circ} \mathrm{C}$. The strain relatedness was calculated with the BioNumerics Tree and Network Inference Module (version 7.6) using band-based Dice similarity coefficient and the unweighted pairs geometric-matched analysis dendogram (band matching tolerance $0.5 \%$ and optimization $0.5 \%$ ) in accordance with the Tenover et al. criteria [23]. The cut-off value to define a PFGE cluster was set at $\leq 6$ band differences (corresponding to equal or less than two genetic events) and $76 \%$.

\section{Infection prevention and control analysis}

Relevant clinical and epidemiological data were collected by an infection control nurse. Bacterial isolates and infections were considered as community-acquired if the collection of the specimen or the start of infection occurred on or before the 2nd day of admission. Thereafter, bacterial isolates and infections were defined as hospital-acquired. Transmission analysis was based on epidemiological data (direct room or ward contact, and/ or documented care by the same staff) and genetic data. Proven transmission events were defined as isolation of genetically-related isolates from two patients who were on the same ward at the same time (at least $24 \mathrm{~h}$, patient-to-patient transmission) or in the same room with a maximum time interval of 6 months (room-to-patient transmission). An interval of 6 months was chosen because transmission of $P$. aeruginosa from environmental sources can last over longer periods and can be sporadic [11]. Hospital-acquired infections were classified according to the CDC definitions [24].

\section{Results}

\section{Isolate and patient characteristics}

Sixty two out of 96 non-duplicate MDR $P$. aeruginosa patient isolates were available for further analysis. Molecular analysis confirmed 21 MBL-test- and cloxacillin- test-positive isolates as CPPA as follows: $\operatorname{lla}_{\mathrm{VIM}-1}(n=$ $2)$, $\operatorname{bla}_{\mathrm{VIM}-2}(n=17), \operatorname{bla}_{\mathrm{IMP}-82}(n=1)$ and $b l a_{\mathrm{NDM}-1} /$ bla $_{\text {GES-5 }}(n=1)$ (Fig. 1). Four cloxacillin-test-positive and MBL-test-negative isolates were not confirmed as carbapenemase-producers.

All CPPA showed an extensively drug-resistant (XDR) phenotype (based on the ECDC/CDC scheme; fosfomycin was not included as there are no clinical breakpoints available according to EUCAST [15]). Fifteen out of 21 CPPA were hospital-acquired, 12 of which were from intensive care units and all except one from the tertiary care centre. Six CPPA were community-acquired. However, five out of these six affected patients received health care within the 30 days before diagnosis. Three patients were transferred to our hospital after hospital stays in Serbia $\left(b l a_{\mathrm{NDM}-1}\right)$, Sri Lanka and Cyprus (bla $\left.a_{\mathrm{IMP}-82}\right)$ or Turkey (bla $\left.a_{\mathrm{VIM}-1}\right)$.

More than half of the patients $(n=11)$ were treated in surgical departments (for trauma, burn, colon disease etc.), eight other patients in internal medicine (for heart or pulmonary disease). Most affected patients $(n=15)$ received an antipseudomonal antibiotic therapy (eight patients had more than one antipseudomonal antibiotic agent) within the 7 days before colonization/infection with CPPA as follows: carbapenems $(n=9)$, ciprofloxacin $(n=8)$, piperacillin-tazobactam $(n=6)$, ceftazidime or cefepime $(n=3)$, and colistin $(n=3)$. Relevant clinical and epidemiological data of the 21 patients colonized/infected with carbapenemase-producing $P$. aeruginosa are summarized in Table 1.

\section{Genotyping and transmission analysis}

RAPD revealed two clusters of VIM-2-producing $P$. aeruginosa containing 13 and 2 isolates each (cluster 1 and cluster 2 respectively). PFGE was only able to confirm cluster 1 (PFGE type A); the PFGE patterns of the cluster 2 isolates displayed eight band differences. All other isolates were unrelated to each other.

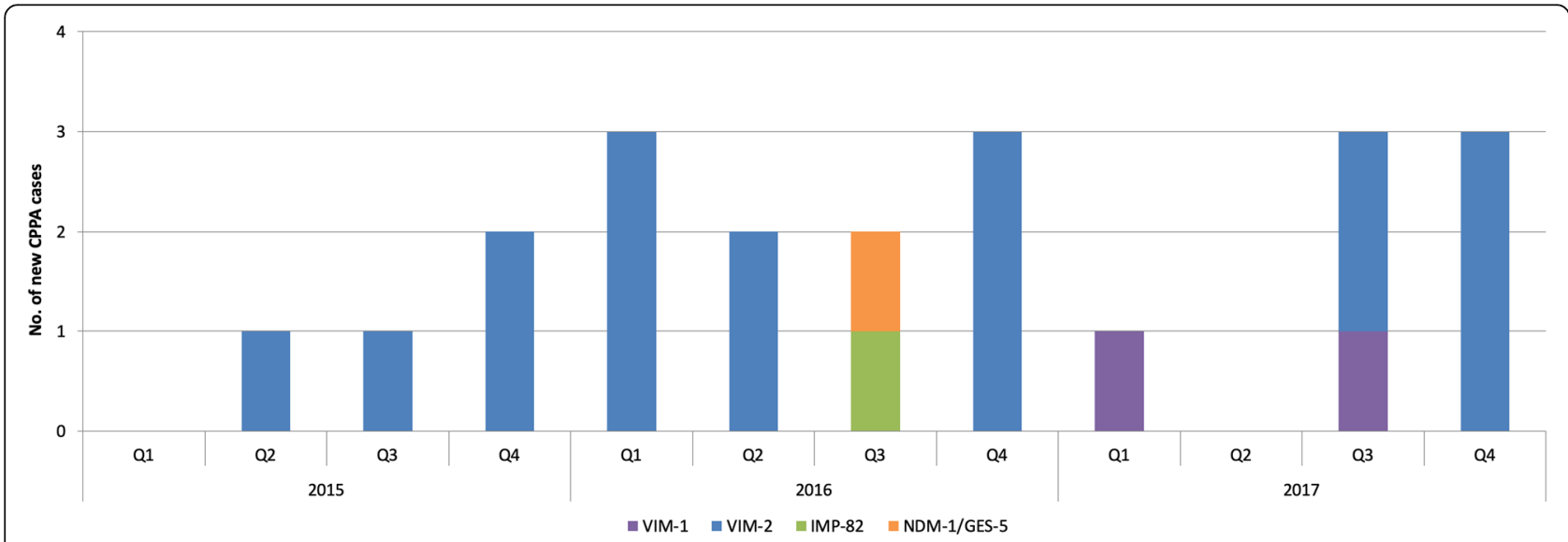

Fig. 1 Overview of new cases with CPPA from 2015 to 2017 (Q = quarter year) 
Table 1 Characteristics of 21 patients with carbapenemaseproducing $P$. aeruginosa

\begin{tabular}{ll}
\hline Patient characteristics $(n=21)$ & Value \\
\hline $\begin{array}{l}\text { Age (years) } \\
\text { mean }\end{array}$ & 62 \\
range & $20 ; 80$ \\
Sex & \\
male & $16(76.2 \%)$ \\
Source of first positive specimen & \\
respiratory tract & $7(33.3 \%)$ \\
urine & $5(23.8 \%)$ \\
screening (rectum) & $5(23.8 \%)$ \\
wound & $2(9.5 \%)$ \\
other & $2(9.5 \%)$ \\
Infection/colonization with CPPA & \\
hospital-acquired & $15(71.4 \%)$ \\
community-acquired & $6(28.6 \%)$
\end{tabular}

Day of acquisition during hospital stay (hospital-acquired CPPA only; $n=15)$

\begin{tabular}{|c|c|}
\hline mean & 19 \\
\hline range & $8 ; 82$ \\
\hline \multicolumn{2}{|l|}{ Medical centres } \\
\hline tertiary care & $20(95.2 \%)$ \\
\hline secondary care & $1(4.8 \%)$ \\
\hline children hospital & $0(0 \%)$ \\
\hline \multicolumn{2}{|l|}{ Ward type } \\
\hline ICU & $14(66.7 \%)$ \\
\hline general ward & $7(33.3 \%)$ \\
\hline \multicolumn{2}{|l|}{ Medical departments } \\
\hline surgery & $11(52.4 \%)$ \\
\hline internal medicine & $8(38.1 \%)$ \\
\hline others & $2(9.5 \%)$ \\
\hline \multicolumn{2}{|l|}{ Hospital-acquired infection (CDC) } \\
\hline pneumonia & $5(23.8 \%)$ \\
\hline urinary tract & $2(9.5 \%)$ \\
\hline skin infection & $2(9.5 \%)$ \\
\hline Antipseudomonal antibiotic therapy ${ }^{a}$ & $15(71.4 \%)$ \\
\hline Surgery $^{a}$ & 15 (71.4\%) \\
\hline Nonsurgical intervention ${ }^{\mathrm{a}}$ & $19(90.5 \%)$ \\
\hline Dialysis $^{a}$ & $6(28.6 \%)$ \\
\hline Mechanical ventilation ${ }^{\mathrm{a}}$ & $16(76.2 \%)$ \\
\hline Wounds $^{\mathrm{a}}$ & $15(71.4 \%)$ \\
\hline Central line $e^{a}$ & $17(80.1 \%)$ \\
\hline Urinary catheter $^{\mathrm{a}}$ & $18(85.7 \%)$ \\
\hline
\end{tabular}

${ }^{a}$ within a maximal interval of 7 days before first isolation of CPPA
Eleven out of 13 PFGE type A isolates were hospitalacquired. However, analysing spatiotemporal links of these patients, we were only able to confirm three patient-to-patient transmissions on three different ICUs (one in 2015 and two in 2017) and one room-to-patient transmission on an ICU in 2017. All transmissions occurred in the tertiary care centre and we were not able to define an index patient as all linked isolates were hospital-acquired (Fig. 2).

\section{Discussion}

In contrast to carbapenem-resistant Acinetobacter baumannii complex or carbapenem-resistant Enterobacterales, carbapenemases are detected less frequently in carbapenem-resistant $P$. aeruginosa in which carbapenemnon-susceptibility is predominantly mediated by other mechanisms (a combination of efflux pumps, AmpC overexpression and porin loss) [5, 25]. However, early detection of these mobile broad-spectrum $\beta$-lactamases is necessary to prevent the propagation mainly of metallo$\beta$-lactamases, across other Gram-negative organisms in the healthcare-setting $[25,26]$.

In our study, carbapenemases, mainly VIM-2, were detected in one third of the MDR/XDR $P$. aeruginosa isolates. The rate of CPPA and proportions of the different carbapenemase gene families in this study are in line with other observations. In 2017 approximately $27.7 \%$ of the $P$. aeruginosa isolates referred to the German reference centre carried a carbapenemase, VIM-2 being by far the most prevalent one [27]. In a German multicentre study, $32 \%$ of the carbapenem-resistant $P$. aeruginosa isolates were carbapenemase producers, with VIM-2 being the most prevalent enzyme [28]. Studies combining molecular surveillance and prevalence data at two German tertiary care centres detected a CPPA proportion of $40 \%$ in MDR isolates (all $b l a_{\mathrm{VIM}}$ ) and $23 \%$ in XDR isolates (mostly bla $a_{\mathrm{VIM}-1}$ and bla ${ }_{\mathrm{VIM}-2}$ ) [29, 30]. Nevertheless, the local epidemiology can differ greatly between medical centres, e.g. in a tertiary care centre $40 \mathrm{~km}$ from Cologne the most prevalent carbapenemase gene in $P$.

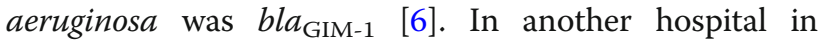
southern Germany bla IMP was widespread [12]. Overall, it is difficult to compare prevalence studies as bacterial isolate selection, inclusion and screening criteria, as well as test algorithms differ greatly. Until now there are no official recommendations by EUCAST addressing carbapenemase screening cut-off values in $P$. aeruginosa comparable to those existing for Enterobacterales [31]. Official screening recommendations are based on the three antibiotics imipenem, meropenem and ceftazidime (German National Reference Centre) or on imipenem, meropenem and piperacillin-tazobactam (British standards) [32, 33]. Overall, we chose a well-defined significant subgroup of MDR $P$. aeruginosa since all isolates 


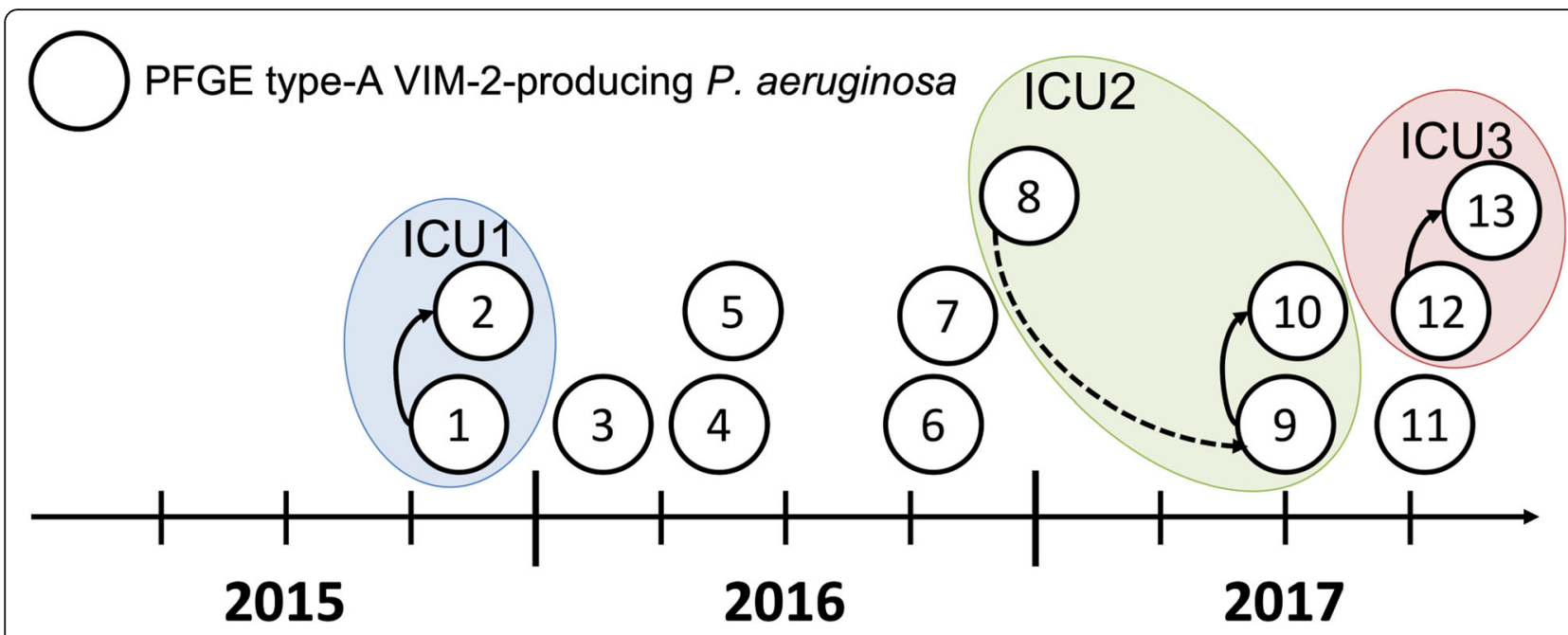

Fig. 2 Epidemiological timeline and transmission route of PFGE type A VIM-2-producing P. aeruginosa. Each node represents one patient at time of first isolation. Arrow indicates genetically and epidemiological confirmed transmission events (dashed line = room-to-patient; continuous line $=$ patient-to-patient). Encircled nodes indicate ward of transmission. Positions of the nodes on the $y$-axis were randomly chosen

non-susceptible to piperacillin, ceftazidime, cefepime, imipenem, meropenem and ciprofloxacin (4MRGN) directly result in infection prevention and control (IPC) measures [14].

Molecular surveillance of bacterial isolates combined with epidemiological and infection data can lead to direct implementation of targeted IPC measures. Surveillance of $P$. aeruginosa is of utmost importance as it can reside in the inanimate patient environment and subsequently lead to transmission and to colonization or infection. $P$. aeruginosa can reside in the sink drains in the patient room for long periods. The spreading and distribution of MDR $P$. aeruginosa in the shower and sink drains, and sewage system of the ward is quite complex as several studies have shown $[11,34]$. We found direct and indirect evidence for both modes of transmission (patient-to-patient and room-to-patient). Although, most bla $_{\mathrm{VIM}-2}$-carrying $P$. aeruginosa isolates clustered in the PFGE analysis, we were only able to confirm a few transmission events. Interestingly, transmission happened exclusively on the intensive care units of the tertiary care centre. Therefore IPC measures should focus on the ICU, where the relevant patients at risk for colonization/ infection with CPPA are found (e. g. antimicrobial therapy, prolonged hospitalization, medical devices, and severe underlying disease) $[2,12,35]$. Moreover, two out of the 13 patients who carried a related (cluster 1) CPPA at admission were referred from another hospital in the region. Thus, genetically related strains may be endemic in the region.

There are a few limitations in this study. We were not able to provide full prevalence data, as only two third non-duplicate 4MRGN isolates detected during this period were available. However, our prevalence data is in line with other studies. Secondly, we were able to detect a dominant bla $a_{\mathrm{VIM}-2}$-carrying strain using PFGE; for further discrimination whole genome sequencing is needed and further studies will address this. Thirdly, our inclusion criteria were probably not sensitive enough to detect all CPPA. On the other hand, CPPA is often associated with MDR- or XDR-phenotypes, corresponding to our inclusion criteria [36]. Extending the screening inclusion criteria would lead to more negative results and clinical microbiology laboratories may not have the resources.

\section{Conclusions}

The surveillance of MDR $P$. aeruginosa based on carbapenemase detection, genotyping and classic epidemiology revealed a relevant prevalence of VIM-2 with endemic spread of a genetically highly-related strains, and proven transmission on intensive care units. This underlines the importance of such methodology for surveillance and the results support the need for a local molecular surveillance system.

\section{Abbreviations}

4MRGN: Multiresistente gramnegative Stäbchen mit Resistenz gegen 4 der 4 Antibiotikagruppen (Gram-negative multidrug-resistant organisms with resistance to 4 antibiotic classes, according to the German classification guideline, see methods); CDC: Centers for Disease Control and Prevention;

CDT: Combined disk test; cgMLST: core genome multilocus sequence type; CPPA: Carbapenemase-producing Pseudomonas aeruginosa; ECDC: European Centre for Disease Prevention and Control; EDTA: Ethylenediaminetetraacetic acid; EUCAST: European Committee on Antimicrobial Susceptibility Testing; ICU: Intensive care unit; IPC: Infection prevention and control; ITSKISS: Intensivstation-Krankenhaus-Infektions-Surveillance-System = German national nosocomial infections surveillance on intensive care units; MALDITOF: Matrix-assisted laser desorption/ionization - time-of-flight mass spectrometer; MBL: Metallo- $\beta$-lactamase; MDR: Multidrug-resistant; PFGE: Pulsed- 
field gel electrophoresis; RAPD: Random amplification of polymorphic DNA; XDR: Extensively drug-resistant

\section{Acknowledgements}

We thank Ingo Winterfeld and the infection control nurse Regine Galante (all from the Institute of Hygiene, Cologne) for technical help.

\section{Authors' contributions}

AFW and FM: Conception and design of the study, acquisition, analysis and interpretation of the data. MM and ES: Acquisition, analysis and interpretation of data. MM, CTC, FM and ES: revising article critically. ES, CTC, NP, LM and PGH: Acquisition of the data. AFW, writing and original draft. All authors read and approved the final manuscript.

\section{Funding}

Parts of this study were performed at the National Reference Centre for multidrug-resistant Gram-negative bacteria. Its work is supported by the Robert Koch Institute with funds provided by the German Ministry of Health (grant no. 1369-402).

\section{Availability of data and materials}

Sequence reads have been deposited at the nucleotide accession number GenBank MN057782. All other data generated or analysed during this study are included in this published article.

\section{Ethics approval and consent to participate}

The study was performed in accordance with the recommendations for surveillance and cluster detections of nosocomial infections of the legally assigned institute for infection control and prevention (Robert Koch Institute). Formal consent was therefore not required.

\section{Consent for publication}

Not applicable.

\section{Competing interests}

The authors declare that they have no competing interests.

\section{Author details}

${ }^{1}$ Institute of Hygiene, Cologne Merheim Medical Centre, University Hospital of Witten/Herdecke, Ostmerheimer Strasse 200, 51109 Cologne, Germany. ${ }^{2}$ Department of Clinical Microbiology, MVZ synlab Leverkusen GmbH, Site Köln-Merheim, Cologne, Germany. ${ }^{3}$ Department of Medical Microbiology, National Reference Centre for Multidrug-resistant Gram-negative Bacteria, Ruhr-University Bochum, Bochum, Germany. ${ }^{4}$ Institute for Medical Microbiology, Immunology and Hygiene, University of Cologne, and German Centre for Infection Research, Partner site Bonn-Cologne, Cologne, Germany.

Received: 5 October 2019 Accepted: 17 December 2019

Published online: 30 December 2019

\section{References}

1. CDC: Centers for Disease Control and Prevention-Antibiotic resistance threats in the United States. 2013.

2. Buhl M, Peter S, Willmann M. Prevalence and risk factors associated with colonization and infection of extensively drug-resistant Pseudomonas aeruginosa: a systematic review. Expert Rev Anti-Infect Ther. 2015;13:1159-70.

3. Wieland K, Chhatwal P, Vonberg RP. Nosocomial outbreaks caused by Acinetobacter baumannii and Pseudomonas aeruginosa: results of a systematic review. Am J Infect Control. 2018;46:643-8.

4. Fochtmann-Frana A, Freystatter C, Vorstandlechner V, Barth A, Bolliger M,

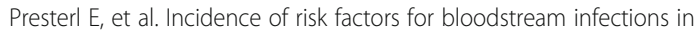
patients with major burns receiving intensive care: a retrospective singlecenter cohort study. Burns. 2018;44:784-92.

5. Poole K. Pseudomonas aeruginosa: resistance to the max. Front Microbiol. 2011;2:65.

6. Wendel AF, Brodner AH, Wydra S, Ressina S, Henrich B, Pfeffer K, et al. Genetic characterization and emergence of the metallo-beta-lactamase GIM-1 in Pseudomonas spp. and Enterobacteriaceae during a long-term outbreak. Antimicrob Agents Chemother. 2013;57:5162-5.

7. Cornaglia G, Giamarellou H, Rossolini GM. Metallo-beta-lactamases: a last frontier for beta-lactams? Lancet Infect Dis. 2011;11:381-93.
8. Walsh TR. Emerging carbapenemases: a global perspective. Int J Antimicrob Agents. 2010;36(Suppl 3):S8-14.

9. Botelho J, Grosso F, Quinteira S, Brilhante M, Ramos H, Peixe L. Two decades of blaVIM-2-producing Pseudomonas aeruginosa dissemination: an interplay between mobile genetic elements and successful clones. J Antimicrob Chemother. 2018;73:873-82.

10. Castanheira M, Deshpande LM, Costello A, Davies TA, Jones RN. Epidemiology and carbapenem resistance mechanisms of carbapenemnon-susceptible Pseudomonas aeruginosa collected during 2009-11 in 14 European and Mediterranean countries. J Antimicrob Chemother. 2014;69:1804-14.

11. Wendel AF, Kolbe-Busch S, Ressina S, Schulze-Robbecke R, Kindgen-Milles D, Lorenz C, et al. Detection and termination of an extended low-frequency hospital outbreak of GIM-1-producing Pseudomonas aeruginosa ST111 in Germany. Am J Infect Control. 2015;43:635-9.

12. Willmann M, Bezdan D, Zapata L, Susak H, Vogel W, Schroppel K, et al. Analysis of a long-term outbreak of XDR Pseudomonas aeruginosa: a molecular epidemiological study. J Antimicrob Chemother. 2015;70:1322-30.

13. Schroder C, Schwab F, Behnke M, Breier AC, Maechler F, Piening B, et al. Epidemiology of healthcare associated infections in Germany: nearly 20 years of surveillance. Int J Med Microbiol. 2015;305:799-806.

14. Muller J, Voss A, Kock R, Sinha B, Rossen JW, Kaase M, et al. Cross-border comparison of the Dutch and German guidelines on multidrug-resistant gram-negative microorganisms. Antimicrob Resist Infect Control. 2015;4:7.

15. Magiorakos AP, Srinivasan A, Carey RB, Carmeli Y, Falagas ME, Giske CG, et al. Multidrug-resistant, extensively drug-resistant and pandrug-resistant bacteria: an international expert proposal for interim standard definitions for acquired resistance. Clin Microbiol Infect. 2012;18:268-81.

16. Pitout JD, Gregson DB, Poirel L, McClure JA, Le P, Church DL. Detection of Pseudomonas aeruginosa producing metallo-beta-lactamases in a large centralized laboratory. J Clin Microbiol. 2005;43:3129-35.

17. Fournier D, Garnier P, Jeannot K, Mille A, Gomez AS, Plesiat P. A convenient method to screen for carbapenemase-producing Pseudomonas aeruginosa. J Clin Microbiol. 2013;51:3846-8.

18. Juan C, Beceiro A, Gutierrez O, Alberti S, Garau M, Perez JL, et al. Characterization of the new metallo-beta-lactamase VIM-13 and its integron-borne gene from a Pseudomonas aeruginosa clinical isolate in Spain. Antimicrob Agents Chemother. 2008;52:3589-96.

19. Dallenne C, Da Costa A, Decre D, Favier C, Arlet G. Development of a set of multiplex PCR assays for the detection of genes encoding important betalactamases in Enterobacteriaceae. J Antimicrob Chemother. 2010;65:490-5.

20. Junemann S, Sedlazeck FJ, Prior K, Albersmeier A, John U, Kalinowski J, et al. Updating benchtop sequencing performance comparison. Nat Biotechnol. 2013;31:294-6.

21. Zankari E, Hasman H, Cosentino S, Vestergaard M, Rasmussen S, Lund O, et al. Identification of acquired antimicrobial resistance genes. J Antimicrob Chemother. 2012;67:2640-4.

22. Wendel AF, Malecki M, Otchwemah R, Tellez-Castillo CJ, Sakka SG, Mattner F One-year molecular surveillance of carbapenem-susceptible A. baumannii on a German intensive care unit: diversity or clonality. Antimicrob Resist Infect Control. 2018;7:145.

23. Tenover FC, Arbeit RD, Goering RV, Mickelsen PA, Murray BE, Persing DH, et al. Interpreting chromosomal DNA restriction patterns produced by pulsed-field gel electrophoresis: criteria for bacterial strain typing. J Clin Microbiol. 1995;33:2233-9.

24. Horan TC, Andrus M, Dudeck MA. CDC/NHSN surveillance definition of health care-associated infection and criteria for specific types of infections in the acute care setting. Am J Infect Control. 2008;36:309-32.

25. Gniadek TJ, Carroll KC, Simner PJ. Carbapenem-resistant non-glucosefermenting gram-negative bacilli: the missing piece to the puzzle. J Clin Microbiol. 2016:54:1700-10.

26. Gillings MR. Integrons: past, present, and future. Microbiol Mol Biol Rev. 2014;78:257-77.

27. Pfennigwerth N. Bericht des Nationalen Referenzzentrums (NRZ) für gramnegative Krankenhauserreger - Zeitraum 1. Januar 2017-31. Epidemiologisches Bull. 2017;2018:263-7.

28. Kresken MK-I, Korte-Berwanger MB, Pfennigwerth N, Gatermann SG. Dissemination of carbapenem-resistant, carbapenemase-non-producing and carbapenemase-producing Pseudomonas aeruginosa in Germany. Eur Congress Clin Microbiol Infect Dis (ECCMID). 2018. O0124. 
29. De Rosa A, Mutters NT, Mastroianni CM, Kaiser SJ, Gunther F. Distribution of carbapenem resistance mechanisms in clinical isolates of XDR Pseudomonas aeruginosa. Eur J Clin Microbiol Infect Dis. 2019;38(8):1547-52.

30. Katchanov J, Asar L, Klupp EM, Both A, Rothe C, Konig C, et al. Carbapenemresistant Gram-negative pathogens in a German university medical center: Prevalence, clinical implications and the role of novel beta-lactam/betalactamase inhibitor combinations. PLoS One. 2018;13:e0195757.

31. EUCAST. EUCAST guidelines for detection of resistance mechanisms and specific resistances of clinical and/ or epidemiological importance, version 2.0. http://www.eucast.org/fileadmin/src/media/PDFs/EUCAST_files/Resistance_ mechanisms/EUCAST_detection_of_resistance_mechanisms_170711.pdf. Accessed 23 Sept 2019.

32. PHE. UK Standards for Microbiology Investigations: Detection of bacteria with carbapenem-hydrolysing $\beta$-lactamases (carbapenemases). https:// assets.publishing.service.gov.uk/government/uploads/system/uploads/ attachment_data/file/554654/B_60i2.1.pdf. Accessed 23 Sept 2019.

33. NRZ. German National Reference Centre for Multidrug-resistant Gramnegative Bacteria: Screening inclusion criteria http://memiserf.medmikro. ruhr-uni-bochum.de/nrz/leistungsspektrum_nrz_carbapenemase-detektion. html. Accessed 23 Sept 2019.

34. Hopman J, Meijer C, Kenters N, Coolen JPM, Ghamati MR, Mehtar S, et al. Risk assessment after a severe hospital-acquired infection associated with Carbapenemase-producing Pseudomonas aeruginosa. JAMA Netw Open. 2019:2:e187665.

35. Zavascki AP, Barth AL, Gaspareto PB, Goncalves AL, Moro AL, Fernandes JF, et al. Risk factors for nosocomial infections due to Pseudomonas aeruginosa producing metallo-beta-lactamase in two tertiary-care teaching hospitals. J Antimicrob Chemother. 2006;58:882-5.

36. Ruiz-Garbajosa P, Canton R. Epidemiology of antibiotic resistance in Pseudomonas aeruginosa. Implications for empiric and definitive therapy. Rev Esp Quimioter. 2017;30(Suppl 1):8-12.

\section{Publisher's Note}

Springer Nature remains neutral with regard to jurisdictional claims in published maps and institutional affiliations.

Ready to submit your research? Choose BMC and benefit from:

- fast, convenient online submission

- thorough peer review by experienced researchers in your field

- rapid publication on acceptance

- support for research data, including large and complex data types

- gold Open Access which fosters wider collaboration and increased citations

- maximum visibility for your research: over $100 \mathrm{M}$ website views per year

At $\mathrm{BMC}$, research is always in progress.

Learn more biomedcentral.com/submissions 